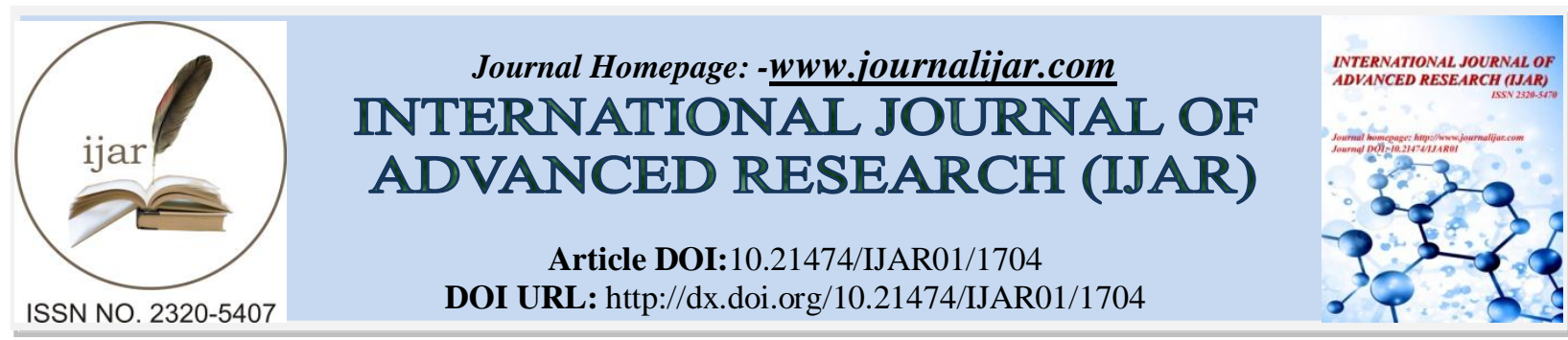

RESEARCH ARTICLE

\title{
MULTIDRUG-RESISTANT ACINETOBACTERBAUMANNII IN ADJARA REGION.
}

\section{Koiava $\mathrm{T}^{1}$, Goncalves $\mathrm{D}^{2,3}$, Palmeira $\mathrm{J}^{2}$, ArobelidzeK. ${ }^{4}$, TavadzeV ${ }^{4}$, Tediashvili $\mathbf{M}^{5}$, Akhvlediani $\mathrm{L}_{.}{ }^{1}$ and Ferreira $\mathbf{H}^{2}$.}

1. Batumi ShotaRustaveli State University, Faculty of Natural Sciences and Health Care-Department of Biology.

2. University of Porto - Pharmacy Faculty - Department of Microbiology.

3. Health Superior Institute of Alto Ave.

4. National Center for Disease Control and Public Healthin Adjara.

5. Eliava Institute of Bacteriophage.

\section{Manuscript Info}

Manuscript History

Received: 14 July 2016

Final Accepted: 19 August 2016

Published: September 2016

Keyword:-

Multidrug-resistant, Acinetobacter baumannii, blaOXA51

\section{Abstract}

Background:- Carbapenem resistance in A. baumannii is now an emerging issue worldwide. Hospital outbreaks have been described from various geographic areas and this organism has become endemic in some of them. Emergence and spread of multidrug-resistant (MDR) Acinetobacter baumannii in nosocomial settings has become a serious concern in clinical practice. Acinetobacter baumannii has multidrug-resistant phenotypes. Resistance to broad spectrum $\beta$ lactams, aminoglycosides, fluoroquinolones, and carbapenems are observed in this bacteria, which complicate the treatment of this pathogen

Objectives: The current study aimed to identify resistance isolate Acinetobacter baumannii from different wards of a teaching hospital in Georgia, Adjara and determine the susceptibility pattern of these bacteria.

Materials and Methods:- Susceptibility profile and identification of the infection Acinetobacter baumannii $(\mathrm{n}=14)$ isolates collected in different hospital services (2013-2015) were performed by disc diffusion methods according to the CLSI guidelines, and API 20E, respectively. Disk diffusion method was employed to evaluate antimicrobial susceptibility against $\boldsymbol{C T X}$-cefotaxime, PIP-Piperacilin, DOR-Doripenem, TIC-Ticarcilin, MRP-Meropenem, FEP-Cefepim, CAZ-Ceftazodom, IMI-Imipenem, TIC/ACC-Ticarcilin/Clavulonic, CIP-ciprofloxacin, STX-Sulfonamid+timetri, CN-Gentamicin, TETetraciclin, TOB-Tobramicin, PIP/TAZ-Piperacilintazobactam, NET-Netilmicin, CT-Colistin and AK-amikacin. Genes of family's blaOXA51, blaOXA40, blaOXA58 and blaOXA23 group were investigated by PCR. Sequencing was performed using group-specific primers

Results:- Among the 14 isolated A. baumannii, Samples cultured from the Sputum (25\%), Biological fluid (17\%),.Most of the isolates (60\%) were obtained from intensive care unit (ICU). Isolated $A$. baumannii showed high resistance to the evaluated antibiotics except

Corresponding Author:-Koiava T.

Address:-Batumi Shota Rustaveli State University, Faculty of Natural Sciences and Health Care- 
TE-Tetraciclin, which showed only $21.4 \%$ resistance. Also, $78.57 \%$ and $100 \%$ of the isolates were identified as MDR.

Conclusions:- The result of the current study showed the growing number of nosocomial infections associated with XDR A. baumannii causing difficulties in antibiotic therapy. Resistant strains increasingly cause public health problems; therefore, their early detection is essential for healthcare centers.

Copy Right, IJAR, 2016,. All rights reserved.

\section{Introduction:-}

Carbapenem resistance in A. baumannii is now an emerging issue worldwide [Krol...2009].Hospital outbreaks have been described from various geographic areas [Landman...2002] and this organism has become endemic in some of them. Emergence and spread of multidrug-resistant (MDR) Acinetobacter baumannii in nosocomial settings has become a serious concern in clinical practice. The organism commonly targets critically ill patients in intensive care units (ICU) and burn wards [Peleg...2008] There is a wide variety of clinical manifestations of Acinetobacter baumannii infections, including hospital-acquired pneumonia, bloodstream infection, urinary tract infection, meningitis and wound infections .ver the last decade, Acinetobacter baumannii has become a serious and emerging nosocomial pathogen worldwide [Peleg...2008]

Acinetobacter baumannii has multidrug-resistant phenotypes. Resistance to broad spectrum $\beta$-lactams, aminoglycosides, fluoroquinolones, and carbapenems are observed in this bacteria, which complicate the treatment of this pathogen [J Coelho...2004].

Control of multidrug-resistant and extensively drug-resistant Acinetobacter spp. infections is an important challenge for clinical microbiologists and physicians. Its ability to survive in hospital environment and its capability to persist for long periods of time on surfaces make it a common cause of healthcare-associated infections and multiple outbreaks [PE Fournier...2006]. The prevalence of A. baumanniiin healthcare centers has increased around the world (Ahmed...2010; Cisneros...2005].

Nevertheless, the most widespread carbapenemases in A. baumannii are class D $\beta$-lactamases. Three main acquired carbapenem-hydrolysing class D oxacillinase (CHDL) gene clusters have been identified either in the chromosome or in plasmids of A. baumannii strains, represented by the blaOXA-23-, blaOXA-24/40-, and blaOXA-58-like genes [Poirel...2006]The OXA-type carbapenemases comprise four broad groups: blaOXA-23-like, blaOXA-40-like, blaOXA-58-like and an intrinsic blaOXA-51-like [Tsakris...2006; Cicek...2014; Kusradze...2011].

Nosocomial outbreaks of imipenem (IPM)-resistant $A$. baumannii producing these OXA enzymes have been reported worldwide: OXA-24-like (OXA-24, OXA-25, OXA-26 and OXA-40) were found in Spain, Belgium, Portugal, Czech Republic, France and the USA; OXA-23-like (OXA-23, OXA-27 and OXA-49) were identified from Europe, Singapore, China, Brazil, Australia, USA, Algeria, Egypt, Libya, South Africa, Thailand, Tunisia, South Korea, Colombia, Iraq and French Polynesia; and OXA-58-like were identified in France, Spain, Belgium, Turkey, Italy, Austria, Greece, the UK, Argentina, Australia, the USA, Kuwait and Pakistan [Peleg,...2008; .Mugnier...2010]Blaoxa-51-like genes are endogenous and specific to A. baumannii. [Woodford...2006; Brown...2005]

\section{Objectives:-}

The current study aimed to identify Acinetobacterbaumannii by molecular method and determine its separation among different wards in hospital and determine the antimicrobial patterns of these bacteria.

\section{Materials and Methods:-}

\section{Antimicrobial susceptibility testing:-}

Disk diffusion method was performed to test the susceptibility of Acinetobacterbaumanniiisolates to common antibiotics on Mueller-Hinton agar, with an inoculum equal to $0.5 \mathrm{McFarland}$ turbidity according to CLSI [16]. The plates were incubated at $37^{\circ} \mathrm{C}$ for $18-24 \mathrm{hrs}$. And the inhibition zone diameters around the antibiotic discs were measured. There were samples of sputum and biological fluids. All isolates were examined for the antibiotic 
resistance of the following antibiotics: CTX-cefotaxime, PIP-Piperacilin, DOR-Doripenem, TIC-Ticarcilin, MRPMeropenem, FEP-Cefepim, CAZ-Ceftazodom, IMI-Imipenem, TIC/ACC- Ticarcilin/Clavulonic, CIPciprofloxacin, STX-Sulfonamid+timetri, CN-Gentamicin, TE-Tetraciclin, TOB-Tobramicin, PIP/TAZPiperacilintazobactam, NET-Netilmicin, CT-Colistin and AK-amikacin.were placed around an Ticarcilinclavulanic acid disc $(85 \mathrm{mg}$ ) at interdisc distances (centre to centre) of $20 \mathrm{~mm}$ on Muller-Hinton agar inoculated by bacterial suspension equal to $0.5 \mathrm{McFarland}$. (Picture 1)



Picture 1:-Acinetobacterbaumanniii-Antimicrobial susceptibility testing.

\section{Imipenem-EDTA synergy test:-}

EDTA(ethylene-diamine-tetraaceticacid) is a polyaminocarboxylicacid that binds metalions like zinc and can in activate the metallo-beta-lactamases. Therefore, it is used for the phenotypic detection of MBL production in clinical isolates (Pitout, 2007)Picture 2.

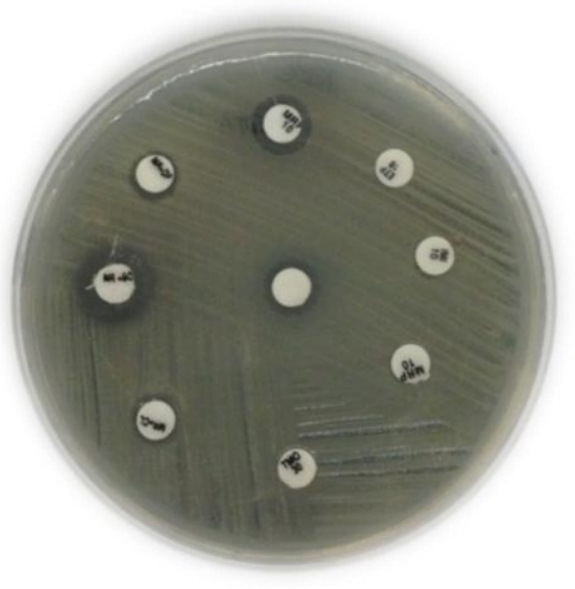

Picture 2:- EDTA synergy test-Acinetobacterbaumanniii..

\section{PCR of Blaoxa-51-like Gene:-}

PCR- Polymerase chain reaction technique has been used of blaOXA51, blaOXA40, blaOXA58 and blaOXA23Genes to confirm the species of $A$. baumannii, genomic DNA of all A. baumannii isolates, PCR was conducted to identify blaoxa-51 genes, which was endogenous to A. baumannii. With specific forward and reverse primers. Table 1.

The PCR conditions were as follows: initial denaturation at $95^{\circ} \mathrm{C}$ for five minutes followed by 35 cycles of $94^{\circ} \mathrm{C}$ for 30 seconds, $52^{\circ} \mathrm{C}$ for 30 seconds, and $72^{\circ} \mathrm{C}$ for 45 seconds and then $72^{\circ} \mathrm{C}$ for 10 minutes. Reactions were performed with $2 \mu$ DNA template.. PCR products were analyzed by electrophoresis on $1.2 \%$ agarose gel in a tris-borate- 
EDTA buffer (TBE) buffer at 85 volts. PCR amplicon size was calculated by comparison to molecular weight size marker (1000 bp DNA ladder). Then the PCR products were visualized under UV light [Safar...2013].

Table 1:-Primers of Acinetobacterbaumanniii

\begin{tabular}{|c|c|c|}
\hline Gene & Primers (5'- 3') & Condition PCR \\
\hline \multirow{2}{*}{$\begin{array}{l}b_{23} \\
\text { OXA- }\end{array}$} & 5'-ATGAATAAATATTTTACTTGC-3' & \\
\hline & 5'- TTAAATAATATTCAGCTGTTT-3' & \\
\hline \multirow{2}{*}{$\begin{array}{l}\text { bla } \\
40\end{array}$} & 5'- ATGAAAAAATTTATACTTCC-3' $^{\prime}$ & \multirow{7}{*}{$\begin{array}{l}\text { Initialdenaturationat } 94 \mathrm{\epsilon} \text { C } 7 \mathrm{~min} \text {; denaturation } \\
\text { at } 94 \mathrm{\epsilon}-40 \mathrm{~s}, \\
\text { annealingat } 57 \mathrm{\epsilon}-40 \mathrm{~s} \\
\text { elongationat } 72 \mathrm{\epsilon}-1 \mathrm{~m} \text {, } \\
\text { repeated for } 30 \text { cycles; } \\
\text { Finalextensionat } 72 \mathrm{\epsilon}-7 \text { minutes }\end{array}$} \\
\hline & 5'- GGTCTACAKCCMWTCCCCA-3' & \\
\hline \multirow{5}{*}{$\begin{array}{l}\text { bla } a_{\mathrm{OXA}-} \\
51 \\
b^{\prime} a_{\mathrm{OXA}-} \\
58\end{array}$} & 5'- ACAGAARTATTTAAGTGGG-3' & \\
\hline & 5'- GGTCTACAKCCMWTCCCA-3' & \\
\hline & 5'- ATGAAATTATTAAAAATATTGAGTTTAG-3' & \\
\hline & 5'- TTATAAATAATGAAAAACACCCAAC-3' & \\
\hline & 5'- AACCCACGATGTGGGTAGC-3' & \\
\hline
\end{tabular}

Figure 1. PCRamplification_-negativecontrol,+1,+5-positivecontrolof blaOXA51 geneof Acinetobacter baumanniiisolates.

All the samples of Acinetobacter baumannii contains carbapenem blaoxa 51 gene. These data are similar to those conducted worldwide research [Woodford ... 2006; Brown ... 2005], including studies conducted in Georgia [Kusradze ... 2011]. The sample of a wounded soldier during the war in Iraq in 2007, containing blaoxa 51 gene,as well as blaoxa 23 gene, has been described in the article written by Kusradze. The sample of phlegm, taken by a wounded soldier being treated at the military hospital of Gori during the Georgian -Russian war in 2008, was studied in 2009. The sample was considered nosocomial, and it contained blaoxa24 gene and as well as blaoxa51 gene, but as the result of our study, conducted in some hospitals in Adjara in 2013-2015, the samples contained blaoxa51 and blaoxa40 genes. (Figure 1, Table 1)Like data of Kusradze, blaOXA-58 was not detected in any isolates in this study.

Table 1. Acinetobacterbaumannii-Sample which contains blaoxa51 and blaoxa40

\begin{tabular}{|c|l|c|}
\hline Sample & Oxa51 & Oxa40 \\
\hline 110 & oxa51 & oxa40 \\
\hline 10 & oxa51 & oxa40 \\
\hline 55 & oxa51 & oxa40 \\
\hline 192 & oxa51 & \\
\hline 5 & oxa51 & \\
\hline 119 & & oxa40 \\
\hline 193 & oxa51 & oxa40 \\
\hline 96 & oxa51 & oxa40 \\
\hline 189 & oxa51 & oxa40 \\
\hline 11 & oxa51 & oxa40 \\
\hline 207 & oxa51 & oxa40 \\
\hline 215 & oxa51 & oxa51 \\
\hline $203-H$ & & \\
\hline
\end{tabular}




\section{Results:-}

Fourteen Acinetobacter baumanniiproducing blaOXA51, blaOXA40, blaOXA58 and blaOXA23gene isolates were detected in different biological samples, namely in sputum $(n=10)$, blood $(n=1)$ and abdominal fluid $(n=3)$, collected in different hospital services. The infection isolates showed an extended resistance profile to aminoglycosides, fluoroquinolones and tetracycline. Isolates showed specific amplification for blaOXA51, blaOXA40, families. AsthediagramshowsallsamplewasresistancetheantibioticsCTX-cefotaxime, PRLPiperacilin, TIC-Ticarcilin, CAZ-Ceftazodom, CT-Colistin, TIC/ACC- Ticarcilin/Clavulonic, tazobactam,., Also quite high rate of resistance to antibiotics STX-Sulfonamid+timetri, CIP-ciprofloxacin, CN-Gentamicin, , TOB-Tobramicin PIP/TAZ-Piperacilin, NET-Netilmicin, and AK-amikacin DORDoripenem, MRP-Meropenem, FEP-Cefepim, IMI-Imipenem,. Relativelysensitiveantibioticswas TETetraciclin.



Figure 2 . Profile of Antibiotic Resistance AcinetobacterBaumannii

Acinetobacter baumannii showed a high resistance to almost all antibiotics. As the diagram shows the highest resistance was revealed towards ceftazidim, it was $\mathbf{1 0 0 \%}$, towards ciprofloxacin, piperacillin, tazobactam, imipenem and ticarcilin / clavulanic $\mathbf{- 9 0 \%}$, towards gentamicin, amikacin, tobramycin, colistin and cfepim $\mathbf{8 0 \%}$. Itshould be noted that our study was different from the research conducted in Georgia. In particular, according to the article „Molecular detection of OXA carbapenemase genes in multidrug-resistant Acinetobacterbaumannii isolates from Iraq and Georgia“ [ Kusradze...2011], all the samples were susceptible 
to colistin, but as the result of our study, $\mathbf{8 0 \%}$ of the samples were resistant to colistin. In general, the resistance profile to broad spectrum antibiotics was similar. The conducted studies showed that the resistance to sulfamet+trimetrop was $\mathbf{7 0} \%$, and a relatively low resistance to tetracycline was $20 \%$ (Figure 2).

\section{Discussion:-}

Acinetobacter spp. is the second most commonly isolated non-fermenter in human specimens (after Pseudomonas aeruginosa). Acinetobacter spp. appears to be an important cause of ICU infections. Multidrug-resistant Acinetobacter spp. is alert pathogens, mostly in ICUs and is related with outbreaks of infection. Almost similar results were observed in a study by Sana Islahi in India. Most of the strains were highly resistant to the antibiotics. Therefore, treatment of these infections are complicated. Evidence has accumulated that contaminated surfaces cause the epidemic and endemic transmission of many MDR and XDR bacteria. [Hossien...2014].

Our results showed that this group of antibiotics had low-level resistance tetracyclin20\%, highest resistance was revealed towards ceftazidim, it was $\mathbf{1 0 0 \%}$, towards ciprofloxacin, piperacillin, tazobactam, imipenem and ticarcilin / clavulanic $\mathbf{- 9 0 \%}$, towards gentamicin, amikacin, tobramycin, colistin and cfepim - 80\%, The conducted studies showed that the resistance to sulfamet+trimetrop was $\mathbf{7 0} \%$. Also, Isolates showed specific amplification for blaOXA51, blaOXA40, families.

In summary, our results demonstrate the need for effective surveillance of antimicrobial resistance in A. baumannii in Adjara Region and suggest that it is essential to use antibiotics with the most caution to prevent the emergence of drug-resistant strains. Furthermore, these findings indicate that the prevalence of antibiotic-resistant A. baumanniiis high in Adjara Region, especially for the antibiotics of choice. This is an emerging concern to public health, particularly in the clinical management of persons with life-threatening A. baumannii infections. The results of this study confirm what some other studies have shown, that the length of hospital stay and antibiotic use prior to infection are significantly associated with increased risk of an antimicrobial resistant $A$. baumanniiinfection

\section{Acknowledgements:-}

Authors thank the staff of Faculty of pharmacy in Porto, Microbiologylaboratory groupof UniversityPorto for their supports.

\section{Referenc:-}

1. Krol V, Hamid NS, Cunha BA. Neurosurgically related nosocomial Acinetobacterbaumannii meningitis: report of two cases and literature review. J Hosp Infect. 2009 Feb. 71(2):176-80. [Medline].

2. Landman D, Quale JM, Mayorga D, Adedeji A, Vangala K, Ravishankar J, et al. Citywide clonal outbreak of multiresistantAcinetobacterbaumannii and Pseudomonas aeruginosa in Brooklyn, NY. Arch Intern Med. 2002;162:1515-20

3. Peleg AY, Seifert H, Paterson DL. Acinetobacterbaumannii: emergence of a successful pathogen. ClinMicrobiol Rev. 2008;21(3):538-82. doi: 10.1128/CMR.00058-07. [PMC free article] [PubMed] [Cross Ref]

4. A.Y. Peleg, H. Seifert, D.L. Paterson Acinetobacterbaumannii: emergence of a successful pathogen ClinMicrobiol Rev, 21 (2008), pp. 538-582

5. . Poirel L, Nordmann P (2006) Carbapenem resistance in Acinetobacterbaumannii: mechanisms and epidemiology. ClinMicrobiol Infect 12: 826-836.

6. IaKusradze ${ }^{\mathrm{a}}$, Seydina M. Diene ${ }^{\mathrm{b}}$, Marina Goderdzishvili ${ }^{\mathrm{a}}$, Jean-Marc Rolain ${ }^{\mathrm{b}}$ Molecular detection of OXA carbapenemase genes in multidrug-resistant Acinetobacterbaumannii isolates from Iraq and Georgia. International Journal of Antimicrobial Agents, Volume 38, Issue 2, August 2011, Pages 164-168

7. PE Fournier, H Richet . The epidemiology and control of Acinetobacterbaumannii in health care facilities. Clin Infect Dis. 2006;42:692-9.

8. Ahmed Hamouda, Benjamin A. Evans, Kevin J.Towner, Sebastian G. B. Amyes . Characterization of Epidemiologically Unrelated Acinetobacterbaumannii Isolates from Four Continents by Use of Multilocus Sequence Typing, Pulsed-Field Gel Electrophoresis, and Sequence-Based Typing of blaOXA-51-like Genes. Journal of Clinical Microbiology. 2010.

9. Cisneros, J. Rodriguez-Bano, F. Fernandez-Cuenca, A. Ribera, J. Vila , A. Pascual, et al. Risk-factors for the acquisition of imipenem-resistant Acinetobacterbaumannii in Spain: a nationwide study. Clin. Microbiol. Infect. $2005 ; 11: 874-879$ 
10. . J Coelho, N Woodford, J Turton, D M Livermore . MultiresistantAcinetobacter in the UK: how big a threat? Journal of Hospital Infections. 2004;58:167-169

11. HossienFazeli 1 ; AzadehTaraghian 1,*; RazieKamali 1 ; FarkhondehPoursina 1 ; Bahram Nasr Esfahani 1 ; ShararehMoghim ,Molecular Identification and Antimicrobial Resistance Profile of Acinetobacterbaumannii Isolated From Nosocomial Infections of a Teaching Hospital in Isfahan, Iran" Avicenna J ClinMicrobInfec. 2014 October; 1(3): e21489.

12. N Woodford, M. J. Ellington, J. M. Coelho, M. E. Ward, S.Brown, J. F. Turton., et al. Multiplex PCR for genes encoding prevalent OXA carbapenemases in Acinetobacter spp. Int.J. Antimicrob. Agents. 2006;27::351353. 14. C Héritier, L Poirel, Fournier PE, JM Claverie, D Raoult, P Nordmann . Characterization of the naturally occurring oxacillinase of Acinetobacterbaumannii. Antimicrob Agents Chemother. 2005;49(10):41749.

13. S Brown , H. K. Young, S. G. B. Amyes . Characterisation of OXA- 51, a novel class D carbapenemase found in genetically unrelated clinical strains of Acinetobacterbaumannii from Argentina. Clin. Microbiol Infect. 2005; 11:15-23

14. SafarFarajnia, FatemehAzhari, MohammadYousefAlikhani, MohammadKazemHosseini , AmirPeymani , NasrolahSohrabi . Prevalence of PER and VEB Type Extended Spectrum Betalactamases among Multidrug Resistant Acinetobacterbaumannii Isolates in North-West of Iran . Iran J Basic Med Sci. 2013;16:751-5.

15. P.D. Mugnier, L. Poirel, T. Naas, P. NordmannWorldwide dissemination of the bla $\boldsymbol{a}_{\text {OXA-23 }}$ carbapenemase gene of AcinetobacterbaumanniiEmerg Infect Dis, 16 (2010), pp. 35-40

16. A.Y. Peleg, H. Seifert, D.L. Paterson Acinetobacterbaumannii: emergence of a successful pathogenClinMicrobiol Rev, 21 (2008), pp. 538-582

17. Cicek AC, Saral A, Iraz M, Ceylan A, Duzgun AO, Peleg AY, Sandalli C. OXA-and GES-type beta-lactamases predominate in extensively drug-resistant Acinetobacterbaumannii isolates from a Turkish University Hospital. ClinMicrobiol Infect. 2014;20(5):410-5. View ArticlePubMedGoogle Scholar

18. Tsakris A, Ikonomidis A, Pournaras S, Tzouvelekis LS, Sofianou D, Legakis NJ, Maniatis AN. VIM-1 metallobeta-lactamase in Acinetobacterbaumannii. Emerg Infect Dis. 2006;12(6):981-3.PubMed CentralViewArticlePubMedGoogle Scholar 